\title{
BMJ
}

\section{Body mass index and risk of liver cirrhosis in middle aged UK women: prospective study}

\author{
Bette Liu, senior research fellow Angela Balkwill, statistical programmer Gillian Reeves, statistical \\ epidemiologist Valerie Beral, professor of epidemiology on behalf of the Million Women Study Collaborators
}

Cancer Epidemiology Unit, University of Oxford, Oxford OX3 7LF

Correspondence to: B Liu Bette.Liu@ceu.ox.ac.uk or bliu@george.org.au

Cite this as: $B M J$ 2010;340:c912 doi:10.1136/bmj.c912

\section{ABSTRACT}

Objective To determine the relation between body mass index (BMI) and liver cirrhosis and the contribution that $\mathrm{BMI}$ and alcohol consumption make to the incidence of liver cirrhosis in middle aged women in the UK.

Design Prospective cohort study (Million Women Study). Setting Women recruited from 1996 to 2001 in NHS breast screening centres and followed by record linkage to routinely collected information on hospital admissions and deaths.

Participants 1230662 women (mean age 56 years at recruitment) followed for an average of 6.2 years.

Main outcome measures Relative risk and absolute risk of first hospital admission with or death from liver cirrhosis adjusted for age, recruitment region, alcohol consumption, smoking, socioeconomic status, and physical activity.

Results 1811 women had a first hospital admission with or died from liver cirrhosis during follow-up. Among women with a BMI of 22.5 or above, increasing BMI was associated with an increased incidence of liver cirrhosis: the adjusted relative risk of cirrhosis increased by $28 \%$ (relative risk $1.28,95 \%$ confidence interval 1.19 to 1.38 ; P<0.001) for every 5 unit increase in BMI. Although the relative increase in the risk of liver cirrhosis per 5 unit increase in BMI did not differ significantly according to the amount of alcohol consumed, the absolute risk did. Among women who reported drinking less than $70 \mathrm{~g}$ alcohol per week, the absolute risk of liver cirrhosis per 1000 women over five years was 0.8 ( 0.7 to 0.9 ) for those with a BMI between 22.5 and 25 and 1.0 (0.9 to 1.2) for those with a BMI of 30 or more. Among women who reported drinking $150 \mathrm{~g}$ alcohol or more per week, the corresponding figures were 2.7 (2.1 to 3.4) and 5.0 (3.8 to 6.6).

Conclusions Excess body weight increases the incidence of liver cirrhosis. In middle aged women in the UK, an estimated $17 \%$ of incident or fatal liver cirrhosis is attributable to excess body weight. This compares with an estimated $42 \%$ attributable to alcohol.

\section{INTRODUCTION}

Cirrhosis of the liver is a growing health problem in the United Kingdom, and deaths from this condition are increasing rapidly among both men and women. Alcohol is a well established cause of cirrhosis, and, although increases in alcohol consumption over the past 10 years are likely to have contributed to the observed rise in rates, other factors may also have a role. ${ }^{12}$ Evidence from prospective studies suggests that excess body weight may result in a substantial increase in the risk of death from liver cirrhosis. ${ }^{3}$ As obesity is becoming more prevalent in the UK population, we examined, in a large prospective cohort of middle aged women, the relation between body mass index and the incidence of hospital admissions with liver cirrhosis and deaths from liver cirrhosis and whether the relation is modified by alcohol or by other factors.

\section{METHODS}

\section{Study population}

The Million Women Study is a prospective cohort study of 1.3 million women who were recruited through National Health Service (NHS) breast screening centres in England and Scotland from 1996 to 2001. At recruitment, women completed a questionnaire (available at www.millionwomenstudy.org) asking about their height, weight, alcohol consumption, and smoking, as well as sociodemographic details, reproductive history, and medical history. They were asked to complete re-survey questionnaires at approximately three to four yearly intervals after recruitment into the study to, among other things, update information provided at recruitment. Height and weight were also measured in a randomly selected sample of 3745 women approximately eight years after recruitment.

Study participants are followed up for hospital admissions and deaths through linkage to centrally held computerised health records by using their NHS number (a unique identifier) and other identifiers including date of birth and sex. These linked records include the NHS central registries for deaths, cancers, and emigrations; the hospital episodes statistics in England $^{4}$; and the Scottish morbidity records in Scotland. ${ }^{5}$ The NHS central registries hold records of all registered deaths, including the cause of death and the date of death. The hospital admission databases contain a record of all NHS inpatient admissions from April 1997 in England and January 1981 in Scotland. Within each hospital record, a main diagnosis 
Table 1|Characteristics of study participants at recruitment and follow-up according to body mass index (BMI) category at recruitment. Values are percentages (numbers) unless stated otherwise

\begin{tabular}{|c|c|c|c|c|c|c|c|}
\hline \multirow[b]{2}{*}{ Characteristics } & \multicolumn{6}{|c|}{ BMI category at recruitment } & \multirow[b]{2}{*}{$\begin{array}{c}\text { All women } \\
(n=1230662)\end{array}$} \\
\hline & $\begin{array}{c}<22.5 \\
(n=237619)\end{array}$ & $\begin{array}{l}22.5 \text { to }<25 \\
(n=331480)\end{array}$ & $\begin{array}{c}25 \text { to }<27.5 \\
(n=266795)\end{array}$ & $\begin{array}{l}27.5 \text { to }<30 \\
(n=173498)\end{array}$ & $\begin{array}{c}30 \text { to }<35 \\
(n=156733)\end{array}$ & $\begin{array}{c}\geq 35 \\
(n=64537)\end{array}$ & \\
\hline Mean (SD) measured BMI* & $22.2(2.2)$ & $25.3(2.3)$ & $27.8(2.6)$ & $30.3(2.7)$ & $33.1(3.5)$ & $37.3(4.9)$ & $27.6(4.9) \dagger$ \\
\hline Mean (SD) age (years) & $55.7(4.7)$ & $55.9(4.7)$ & $56.2(4.7)$ & $56.3(4.7)$ & $56.3(4.7)$ & $55.8(4.6)$ & $56.0(4.7)$ \\
\hline In upper third of socioeconomic status & $37.5(88329)$ & $37.2(122$ 267) & $34.4(90970)$ & $31.5(54328)$ & $28.1(43698)$ & $23.3(14927)$ & $33.9(414519)$ \\
\hline Reported drinking alcohol & $79.0(186626)$ & $80.8(266323)$ & $78.1(206963)$ & 74.5 (128 290) & 69.5 (107 981) & $61.2(39101)$ & $76.5(935284)$ \\
\hline Mean (SD) alcohol intake reported by drinkers (g/week) & $59.6(56.8)$ & $57.0(54.9)$ & $53.7(54.0)$ & $50.6(53.4)$ & $46.4(51.9)$ & $40.8(50.1)$ & $54.0(54.6)$ \\
\hline Current smokers & $25.4(57389)$ & $20.6(64758)$ & $19.6(49294)$ & 18.8 (30 599) & $17.1(25050)$ & $15.3(9$ 190) & $20.3(236280)$ \\
\hline Treated for diabetes & $0.84(2005)$ & $1.1(3625)$ & $1.7(4611)$ & $2.9(5,021)$ & $5.0(7821)$ & $9.3(5985)$ & $2.4(29068)$ \\
\hline Doing strenuous physical activity more than once a week & $46.0(105766)$ & $44.4(142579)$ & 39.5 (102 018) & $34.7(58056)$ & $29.6(44709)$ & $23.9(14829)$ & $39.4(467957)$ \\
\hline Mean (SD) person years' follow-up & $6.2(1.2)$ & $6.2(1.2)$ & $6.2(1.2)$ & $6.1(1.2)$ & $6.1(1.2)$ & $6.1(1.2)$ & $6.2(1.2)$ \\
\hline No of events & 414 & 402 & 343 & 236 & 283 & 133 & 1811 \\
\hline
\end{tabular}

and up to 13 additional diagnoses are coded. During the period of follow-up for this study, both the cause of death and diagnoses on admission to hospital were coded by using ICD-10 (the international classification of diseases, version 10). All study participants gave signed consent to be included.

\section{Data definitions}

We classified women as having a hospital admission with liver cirrhosis or death from liver cirrhosis if, during follow-up, they had either a hospital record or a death registration with an ICD10 code of K70, K73, or K74. These ICD10 codes are consistent with those used in other epidemiological studies of liver cirrhosis in the UK. ${ }^{1}$ We calculated body mass index as a woman's weight in kilograms divided by her height in metres squared. Alcohol use was reported as standard drinks consumed on average in a week - that is, number of glasses of wine, half pints of beer/lager, or measures of spirits. We converted this into grams, considering one standard drink to be equivalent to $10 \mathrm{~g}$ of alcohol. $^{6}$

\section{Analysis}

We excluded participants from analyses if they reported having had any type of liver disease (including viral hepatitis) before recruitment, if they had a hospital admission record of any liver disease before recruitment (ICD 10 codes K70-K77, B15-B19), if they had a diagnosis of cancer (except non-melanomatous skin cancer, ICD-10 code C44) before recruitment, or if their body mass index at recruitment was unknown. We used Cox regression models to analyse data. We followed women from the date of recruitment to the date of hospital admission with cirrhosis, the date of death from cirrhosis, or the last date of follow-up, whichever came first. The last date for which we had complete hospital and death data was 31 December 2003 for women recruited in Scotland and 31 March 2005 for those recruited in England. For a small proportion (5\%) of women recruited in England before 1 April 1997, we calculated person years from this date as hospital records were not available in England before this time.

We estimated the risk of hospital admission with cirrhosis or death from cirrhosis for six categories of body mass index $(<22.5,22.5$ to $<25,25$ to $<27.5,27.5$ to $<30,30$ to $<35$, and $\geq 35$ ) at recruitment and also, in those with a value greater than 22.,5 for each 5 unit increase. To correct for misclassification that may result from self reporting of body mass index, ${ }^{7}$ and for changes in body mass index over time, ${ }^{8}$ we took the mean value in each category as that measured in the randomly selected sample of women $(n=3745)$ after recruitment. We used the measured mean value in each category of body mass index in tests of trend across the categories.

As no natural baseline exists for categories of body mass index, we calculated floating absolute risks and set the reference group as women with a body mass index of between 22.5 and 25. Compared with conventional methods, floating absolute risks do not alter the relative risk estimates but reduce the variances attributed to them and permit tests of trend. ${ }^{910}$ In regression analyses, we used attained age as the underlying time variable and routinely stratified all analyses for region of recruitment (10 regions) and adjusted them for alcohol intake in categories (none (including never or past drinkers) and, among drinkers, consumptions of $<30$, 30 to $<70,70$ to $<150$, and $\geq 150 \mathrm{~g} /$ week), socioeconomic status in fifths (according to the deprivation index, a score based on residential address that takes into account information on employment, household overcrowding, and home and car ownership), ${ }^{11}$ smoking status and amount smoked (never, past, current 1-9 cigarettes a day, current 10-19 cigarettes a day, and current $\geq 20$ cigarettes a day), and strenuous physical activity (once a week or less, more than once a week). We also examined the effect of adjustment for additional factors (use of hormonal therapies, parity, year 
Table $2 \mid$ Minimally adjusted and fully adjusted relative risk of cirrhosis related hospital admission and death according to measured mean body mass index (BMI) in BMI category at recruitment

\begin{tabular}{lcc}
$\begin{array}{l}\text { BMI category at recruitment } \\
\text { (mean BMI*) }\end{array}$ & $\begin{array}{c}\text { Minimally adjusted } \\
\text { relative risk } \neq\end{array}$ & $\begin{array}{c}\text { Adjusted relative risk } \\
(95 \% \mathrm{FCl})\end{array}$ \\
\hline $22.5(22.2)$ & 1.46 & $1.36(1.23$ to 1.50$)$ \\
\hline 22.5 to $25(25.3)$ (reference) & 1.00 & $1.00(0.91$ to 1.10$)$ \\
\hline 25 to $<27.5(27.8)$ & 1.05 & $1.05(0.94$ to 1.17$)$ \\
\hline 27.5 to $<30(30.3)$ & 1.11 & $1.11(0.97$ to 1.26$)$ \\
\hline 30 to $<35(33.1)$ & 1.48 & $1.49(1.33$ to 1.68$)$ \\
\hline$\geq 35(37.3)$ & 1.72 & $1.77(1.49$ to 2.10$)$ \\
\hline Per 5 unit increase in BMI§ & 1.27 & $1.28(1.19$ to 1.38$)$ \\
\hline
\end{tabular}

$\mathrm{FCl}=$ floated confidence interval (see methods).

${ }^{\star}$ Calculated using measured height and weight in randomly selected sample of cohort (see methods). †Adjusted for age and region.

$\ddagger$ Adjusted for age, region, socioeconomic status, alcohol consumption, smoking, and physical activity. $\S$ In women with $\mathrm{BMI} \geq 22.5$.

\section{RESULTS}

After exclusions for a diagnosis of cancer before recruitment $(n=44196)$, liver disease before recruitment (2156), or unknown body mass index (67943), we included 1230662 women in the analyses. Over a mean of 6.2 person years of follow-up, 1811 women had a first cirrhosis related hospital admission or death and 421 of these women had cirrhosis recorded for the first time at death. The overall incidence of first hospital admission with or death from cirrhosis in this population was 1.2 per 1000 women over five years.

Table 1 shows the characteristics of study participants according to categories of body mass index reported at recruitment. On the basis of the World Health Organization's definitions for body mass index, $46 \%$ of women in the study were a healthy weight or less (body mass index $<25$ ), 36\% were overweight $(25$ to $<30)$, and $18 \%$ were obese $(\geq 30)$. The mean age of women at recruitment was 56 years, and the mean measured body mass index was 27.6. The proportion of women in the upper socioeconomic group decreased with increasing body mass index. Of the women included in these analyses, 77\% reported drinking some alcohol; among the drinkers, the mean reported alcohol consumption was $54 \mathrm{~g}$ /week or approximately five and a half standard drinks a week. The proportion of women reporting drinking any alcohol and the amount they drank decreased with increasing body mass index. The proportion of women who were current smokers and the proportion who reported doing strenuous physical activity more than once a week also decreased with increasing body mass index. As expected, the proportion who reported being treated for diabetes increased with increasing body mass index.

Figure 1 shows the relative risks for a first hospital admission with or death from cirrhosis according to body mass index. Routine adjustment for socioeconomic status, alcohol consumption, smoking, and physical activity in addition to age and recruitment region did not alter the pattern of risks substantially (table 2). Compared with the reference group (women with a body mass index of 22.5 to $<25$ ), both the women

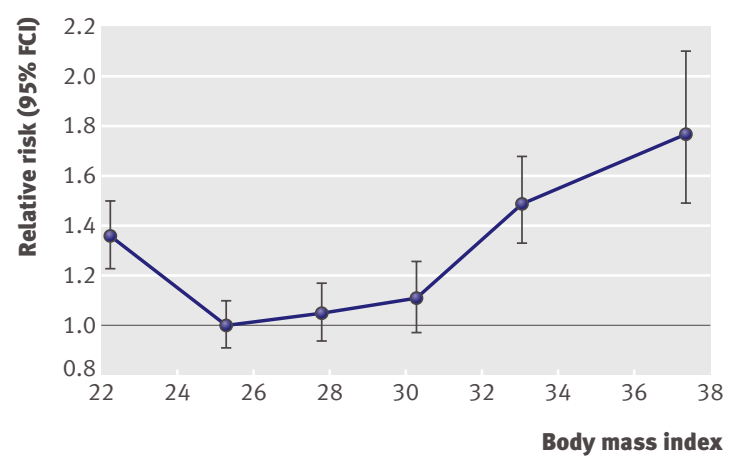

Fig 1| Relative risk of liver cirrhosis according to body mass index. $\mathrm{FCl}=$ floated confidence interval. * Relative risk plotted against mean measured body mass index (BMI) in each BMI category (see methods) 
Table 3 |Sensitivity analysis comparing relative risks of cirrhosis related hospital admission and death according to measured mean body mass index (BMI) in BMI category at recruitment in main analyses, censoring first 2 years of follow-up, and using only mortality data

\begin{tabular}{|c|c|c|c|c|c|c|}
\hline \multirow{2}{*}{$\begin{array}{l}\text { BMI category at recruitment } \\
\text { (mean BMI*) }\end{array}$} & \multicolumn{3}{|c|}{ Cases } & \multicolumn{3}{|c|}{ Relative risk $\ddagger(95 \% \mathrm{FCI})$} \\
\hline & Main analysis & Censoring first 2 years & Mortality data only & Main analysis & Censoring first 2 years & Mortality data only \\
\hline$<22.5(22.2)$ & 414 & 286 & 106 & 1.36 & 1.26 & 1.30 \\
\hline 22.5 to $<25$ (25.3) (reference) & 402 & 297 & 103 & 1.00 & 1.00 & 1.00 \\
\hline 25 to $<27.5(27.8)$ & 343 & 262 & 77 & 1.05 & 1.10 & 0.95 \\
\hline 27.5 to $<30(30.3)$ & 236 & 169 & 52 & 1.11 & 1.09 & 1.01 \\
\hline 30 to $<35(33.1)$ & 283 & 216 & 54 & 1.49 & 1.59 & 1.24 \\
\hline$\geq 35$ (37.3) & 133 & 102 & 29 & 1.77 & 1.91 & 1.81 \\
\hline Per 5 unit increase in BMI† & 1397 & 1046 & 315 & 1.28 & 1.31 & 1.24 \\
\hline
\end{tabular}

$\mathrm{FCl}=$ floated confidence interval (see methods).

*Calculated using measured height and weight in a randomly selected sample of cohort (see methods).

†In women with $\mathrm{BMI} \geq 22.5$.

$\ddagger$ Adjusted for age, region, socioeconomic status, alcohol consumption, smoking, and physical activity.

who had a lower body mass index and those with a higher body mass index had a significantly greater relative risk of cirrhosis. Among women with body mass index below 22.5, we cannot exclude the possibility that previous illness may have contributed to weight loss, and for this reason we focused our analyses on women with a body mass index of 22.5 or above. Among women who had a body mass index of 22.5 or above, little evidence existed to suggest non-linearity in the relation between body mass index and the relative risk of cirrhosis related hospital admission or death (test for non-linearity, $\mathrm{P}=0.2$ ). Among these women, the estimated increase in the risk of cirrhosis was $28 \%$ (95\% confidence interval $19 \%$ to $38 \%$ ) for each 5 unit increase in body mass index.

Table 3 shows results from various sensitivity analyses. When we censored the first two years of followup, 1332 cirrhosis related admissions and deaths occurred over a mean of 4.2 years of follow-up. When we used mortality data only, 421 deaths occurred over a mean of 6.2 years of follow-up. In both scenarios, the relation between body mass index and cirrhosis was similar to that in the main analysis. When we adjusted for additional potential confounders including reproductive history, use of hormonal therapies reported at recruitment, and year of birth (which, together with the routine adjustment for age, adjusts for calendar year during follow-up), the relative risks for the effect of body mass index on liver cirrhosis changed by less than $5 \%$.

We compared the effect of body mass index on the relative risk of cirrhosis in categories of alcohol consumption, smoking, and diabetes reported at recruitment in women with body mass index 22.5 or above (table 4 ). We found that the trend in the relative risk with increasing body mass index did not differ significantly between drinkers with increasingly larger consumptions of alcohol $(<70,70$ to $<150$, or $\geq 150 \mathrm{~g} /$ week $)$ or between women who had diabetes or not (likelihood ratio tests for heterogeneity, $\mathrm{P}=0.7$ and $\mathrm{P}=0.1$ ). The relative risk of cirrhosis with increasing body mass index did, however, differ according to whether women were current smokers or not (test for heterogeneity, $\mathrm{P}<0.001$ ). Current smokers had relative risks of cirrhosis almost three times those of never smokers, but we found little or no trend of increasing risk with increasing body mass index among current smokers.

Figure 2 shows the rates of liver cirrhosis per 1000 women in this cohort over five years. The rates increase both with body mass index and with the amount of alcohol consumed. Although the relative risk per unit increase in body mass index does not vary by alcohol intake, the absolute risk does. In women drinking less than $70 \mathrm{~g}$ alcohol per week (mean intake 0.4 drinks/day), the incidence of liver cirrhosis was 0.8 (95\% confidence interval 0.7 to 0.9$)$ per 1000 over five years for those with body mass index between 22.5 and 25 and increased to 1.0 (0.9 to 1.2 ) per 1000 in women with body mass index 30 or above, whereas the corresponding figures for women drinking $150 \mathrm{~g}$ or more per week (mean intake 2.5 drinks per day) were 2.7 (2.1 to 3.4) and 5.0 (3.8 to 6.6) per 1000 .

Data from population based surveys in the UK indicate that the average alcohol intake in women is $9.3 \mathrm{~g} /$ day and that approximately $31 \%$ do not drink alcohol ${ }^{16}$; the average intake among drinkers is thus $13.5 \mathrm{~g} /$ day. The distribution of body mass index in middle aged women in the UK is $13 \%$ with a body

Reported alcohol consumption

- - $<70 \mathrm{~g} /$ week $\quad-0-70$ to $<150 \mathrm{~g} /$ week --o- $\geq 150 \mathrm{~g} /$ week

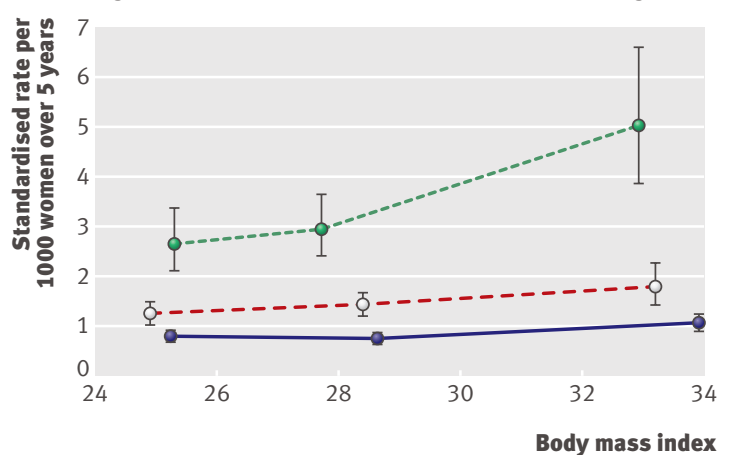

Fig 2 | Standardised rates (with $95 \% \mathrm{Cl}$ ) for liver cirrhosis per 1000 women over 5 years by body mass index (BMI) and alcohol consumption. Rate plotted against mean measured $\mathrm{BMI}$ in each BMI category (see methods) 
Table $4 \mid$ Relative risk of cirrhosis according to body mass index (BMI) in women drinking $<70,70$ to $<150$, and $\geq 150 \mathrm{~g} /$ week alcohol; in never and current smokers; and in women with and without diabetes

\begin{tabular}{|c|c|c|c|c|c|c|c|}
\hline \multirow{2}{*}{$\begin{array}{l}\text { BMI category at } \\
\text { recruitment }\end{array}$} & \multicolumn{3}{|c|}{$\begin{array}{l}\text { Relative risk* }(95 \% \mathrm{FCl}) \text { according } \\
\text { to reported alcohol consumption }\end{array}$} & \multicolumn{2}{|c|}{$\begin{array}{c}\text { Relative risk* }(95 \% \mathrm{FCl}) \text { according } \\
\text { to smoking status }\end{array}$} & \multicolumn{2}{|c|}{$\begin{array}{c}\text { Relative risk* }(95 \% \mathrm{FCl}) \text { according } \\
\text { to diabetes status }\end{array}$} \\
\hline & $<70 \mathrm{~g} /$ week & 70 to $<150 \mathrm{~g} /$ week & $\geq 150 \mathrm{~g} /$ week & Never & Current & No diabetes & Diabetes \\
\hline 22.5 to $<25$ & $\begin{array}{c}1.00 \text { (0.85 to } 1.17) \\
\text { (reference) }\end{array}$ & 1.59 (1.31 to 1.92$)$ & 3.44 (2.70 to 4.37$)$ & $\begin{array}{c}1.00 \text { (0.83 to } 1.20) \\
\text { (reference) }\end{array}$ & 2.68 (2.31 to 3.13$)$ & $\begin{array}{c}1.00 \text { (0.90 to 1.11) } \\
\text { (reference) }\end{array}$ & $4.29(2.74$ to 6.73$)$ \\
\hline 25 to $<30$ & 0.96 (0.84 to 1.10$)$ & 1.83 (1.56 to 2.16$)$ & 3.82 (3.09 to 4.72$)$ & $1.23(1.07$ to 1.41$)$ & 2.47 (2.15 to 2.85$)$ & 1.05 (0.96 to 1.15$)$ & 4.37 (3.30 to 5.78$)$ \\
\hline$\geq 30$ & 1.35 (1.15 to 1.59$)$ & 2.31 (1.81 to 2.94$)$ & 6.53 (4.98 to 8.55$)$ & 1.66 (1.41 to 1.96$)$ & $2.96(2.43$ to 3.62$)$ & 1.38 (1.24 to 1.54$)$ & $5.94(4.83$ to 7.31$)$ \\
\hline
\end{tabular}

$\mathrm{FCl}=$ floated confidence interval (see methods).

${ }^{\star}$ Adjusted for age, region, socioeconomic status, physical activity, and alcohol consumption and smoking as appropriate.

mass index under $22.5,21 \%$ with between 22.5 and 25 , $39 \%$ overweight, and $28 \%$ obese. ${ }^{15}$ On the basis of these figures, we estimate that in middle aged women in the UK, approximately $42 \%$ of hospital admissions with cirrhosis or deaths from cirrhosis can be attributed to alcohol consumption and $17 \%$ to excess body weight (body mass index $\geq 25$ ).

\section{DISCUSSION}

In this study of middle aged women in the UK who consume low to moderate amounts of alcohol, we found that compared with women with a body mass index between 22.5 and 25 , those who were overweight or obese had an increased risk of liver cirrhosis; the risk increased by about $28 \%$ for each 5 unit increase in body mass index. The relative increase in the risk of liver cirrhosis was not altered by alcohol consumption; however, the absolute increase in liver cirrhosis rates with increasing body mass index was substantially greater in women who reported that they drank $150 \mathrm{~g}$ or more of alcohol per week (an average of two and a half drinks a day) than in those reporting drinking less than $70 \mathrm{~g}$ a week (an average of about half a drink a day).

\section{Findings in relation to other studies}

Our findings on the relation between body mass index and liver cirrhosis are broadly consistent with those of previous prospective studies, ${ }^{317}$ but the magnitude of the risk estimate is somewhat lower than that described in a large collaborative reanalysis of data from prospective studies, ${ }^{3}$ The collaborative reanalysis differed in that it included men and women, used mortality from liver cirrhosis as the end point, and excluded the first five years of follow-up. We found that women with a body mass index below 22.5 had a greater relative risk of liver cirrhosis than did those with a body mass index between 22.5 and 25 , and, similar to results from the large collaboration, ${ }^{3}$ this increased risk remained after exclusion of the first few years of follow-up (see table 3). Interpreting the relevance of associations between low body mass index and chronic disease such as cirrhosis is difficult, as analyses may not adequately compensate for the likelihood that early liver disease may affect body mass index before the first hospital admission or death occurs-for example, by reducing appetite or by causing malabsorption. For this reason, we focused on women with a body mass index of 22.5 or above.
Obesity results in an increase in fat deposition within the hepatocytes and the development of fatty liver (hepatic steatosis). This can lead to inflammation (nonalcoholic steatohepatitis) and subsequent liver fibrosis and cirrhosis, ${ }^{18}$ although the mechanisms that determine whether fibrosis and cirrhosis develop are poorly understood. ${ }^{19}$ Fatty liver is also found in people with a high alcohol intake and diabetes, and hepatic steatosis in the presence of diabetes may increase the likelihood of progression to cirrhosis. ${ }^{19}$ We found that the relative risks associated with increasing body mass index did not vary with moderate alcohol intake or with a history of diabetes but did vary by smoking history. The limited literature on smoking and its association with liver cirrhosis makes it difficult to draw conclusions about what factors may underlie this result.

\section{Strengths and limitations}

Owing to the large number of cases in this prospective study, we were able to estimate reliably the association between body mass index and incidence of liver cirrhosis in middle aged women. We were also able to examine whether this association was modified by low to moderate alcohol intake (up to about two and a half drinks a day on average), smoking, and diabetes in women. Only a small proportion of women in this cohort reported consuming more than three drinks a day, so we were unable to look at the effects of heavy alcohol consumption. Viral hepatitis is also an important cause of cirrhosis, but because of the low rates in this study population of predominantly white middle aged UK women, ${ }^{20}$ and the lack of serological evidence to identify participants with viral hepatitis, we could not examine whether the effect of body mass index on cirrhosis is modified by this factor.

We used NHS hospital records and mortality records to ascertain outcomes. Mortality records are used in most epidemiological studies of liver cirrhosis $^{13}$; although the use of hospital admission data is novel for identifying liver cirrhosis events, linkage to hospital data has been shown to be good, ${ }^{5}$ and hospital diagnoses are coded independently of the study investigators. Our sensitivity analyses in which we compared our main findings for deaths only with those for hospital admissions and deaths (table 3) were consistent. 


\section{WHAT IS ALREADY KNOWN ON THIS TOPIC}

Rates of liver cirrhosis and obesity are increasing in the UK

Although alcohol is a major cause of liver cirrhosis, recent evidence suggests that excess body weight may also play a role

\section{WHAT THIS STUDY ADDS}

Excess body weight increases the risk of liver cirrhosis in women

Among middle aged women in the UK, excess body weight contributes to almost $20 \%$ of the cirrhosis related hospital admissions and deaths, and alcohol contributes to almost $50 \%$

Body mass index and alcohol consumption are known to be affected by reporting errors, with some under-reporting of body mass index among more overweight people and some under-reporting of alcohol consumption in heavier drinkers. With respect to body mass index, in order to minimise misclassification, we analysed and presented the results by using values measured subsequently within each category of body mass index. With respect to alcohol consumption, as the women in this study drank mostly low to moderate quantities of alcohol, misclassification may be less likely to affect our findings. To account for regression dilution bias, we calculated trends across categories of alcohol consumption reported at recruitment but assigned the level of intake in each category as the average alcohol consumption reported subsequently. The proportion of women in the Million Women Study who reported drinking more than $150 \mathrm{~g} /$ week of alcohol is less than that estimated from UK population surveys of women in this age range, ${ }^{15}$ and, as mentioned previously, our findings may not apply to heavy drinkers.

\section{Implications}

Our results suggest that compared with the other known risk factors for cirrhosis, the effect of obesity is moderate (relative risk 1.28 per 5 unit increase for women with body mass index $\geq 22.5$ ). In the UK, middle aged women are not heavy consumers of alcohol. ${ }^{16}$ Among women in this study who reported drinking an average of about a third to a half an alcoholic drink a day, we estimated that over five years 0.8 in 1000 with a healthy weight will be admitted to hospital with or will die from liver cirrhosis compared with 1.0 in 1000 women who are obese. However, among women who reported drinking an average of two and a half alcoholic drinks a day, over five years 2.7 in 1000 with a healthy weight will be admitted to hospital with or will die from liver cirrhosis compared with 5.0 in 1000 women who are obese. We cannot estimate the effects of excess body weight on the incidence of liver cirrhosis in heavy consumers of alcohol, or in men; to better understand what factors underlie the rising rates death from liver cirrhosis in the UK, the effects of overweight and obesity in these populations warrant further investigation.

In summary, excess body weight clearly makes an independent contribution to rates of liver cirrhosis, and in middle aged women we estimated this to be about $17 \%$ of all cirrhosis related hospital admissions and deaths, or almost half of the proportion attributable to alcohol. From a public health perspective, reducing both excessive alcohol consumption and excessive body weight should lead to a reduction in the incidence of liver cirrhosis.

We thank all the women who participated in the Million Women Study and the study steering committee, as well as ISD Scotland, the Information Centre for Health and Social Care, and Northgate Solutions for the linkage of hospital records and Adrian Goodill for drawing the figures. Million Women Study Steering Committee: Joan Austoker, Emily Banks, Valerie Beral, Judith Church, Ruth English, Jane Green, Julietta Patnick, Richard Peto, Gillian Reeves, Martin Vessey, and Matthew Wallis. Million Women Study coordinating centre staff: Simon Abbott, Miranda Armstrong, Krys Baker, Angela Balkwill, Vicky Benson, Valerie Beral, Judith Black, Anna Brown, Diana Bull, Benjamin Cairns, James Chivenga, Barbara Crossley, Dave Ewart, Sarah Ewart, Lee Fletcher, Laura Gerrard, Adrian Goodill, Isobel Green, Jane Green, Elizabeth Hilton, Joy Hooley, Sau Wan Kan, Carol Keene, Oksana Kirichek, Nicky Langston, Bette Liu, Maria-Jose Luque, Maria MacGregor, Lynn Pank, Kirstin Pirie, Gillian Reeves, Emma Sherman, Evie Sherry-Starmer, Moya Simmonds, Elizabeth Spencer, Helena Strange, Siân Sweetland, Alison Timadjer, Sarah Tipper, Ruth Travis, Xiaosi Wang, Joanna Watson, Stephen Williams, Lucy Wright, Tienyu Yang, and Heather Young. Collaborating UK NHS breast screening centres: Avon, Aylesbury, Barnsley, Basingstoke, Bedfordshire and Hertfordshire, Cambridge and Huntingdon, Chelmsford and Colchester, Chester, Cornwall, Crewe, Cumbria, Doncaster, Dorset, East Berkshire, East Cheshire, East Devon, East of Scotland, East Suffolk, East Sussex, Gateshead, Gloucestershire, Great Yarmouth, Hereford and Worcester, Kent, Kings Lynn, Leicestershire, Liverpool, Manchester, Milton Keynes, Newcastle, North Birmingham, North East Scotland, North Lancashire, North Middlesex, North Nottingham, North of Scotland, North Tees, North Yorkshire, Nottingham, Oxford, Portsmouth, Rotherham, Sheffield, Shropshire, Somerset, South Birmingham, South East Scotland, South East Staffordshire, South Derbyshire, South Essex, South Lancashire, South West Scotland, Surrey, Warrington Halton St Helens and Knowsley, Warwickshire Solihull and Coventry, West Berkshire, West Devon, West London, West Suffolk, West Sussex, Wiltshire, Winchester, Wirral, and Wycombe.

Contributors: BL conceived the study, specified the analyses, and wrote the manuscript. $A B$ did the analyses and edited drafts of the manuscript. GR specified the analyses and edited drafts of the manuscript. VB conceived the study, specified analyses, and edited drafts of the manuscript. VB and BL are the guarantors.

Funding: This study was funded by Cancer Research UK, the NHS Breast Screening Programme, and the Medical Research Council. The funders did not participate in the study design and conduct or in the collection, management, analysis, and interpretation of the data, nor did they have any input into the preparation, review, or approval of this manuscript. Competing interests: None declared.

Ethical approval: The Million Women Study has been approved by the Eastern Multi-centre Research Ethics Committee, and all study participants gave signed consent to be included. Access and linkage to hospital records was approved by the Information Centre for Health and Social Care in England and the Information and Statistics Division in Scotland.

Data sharing: All information provided is stored in accordance with the Data Protection Act (Office of Data Protection Registrar registration No K3039784). Only the study team has access to computerised data, via passwords (see the Million Women Study protocol at www. millionwomenstudy.org).

1 Leon DA, McCambridge J. Liver cirrhosis mortality rates in Britain from 1950 to 2002: an analysis of routine data. Lancet 2006;367:52-6.

2 Williams JG, Roberts SE, Ali MF, Cheung WY, Cohen DR, Demery G, et al. Gastroenterology services in the UK: the burden of disease, and the organisation and delivery of services for gastrointestinal and liver disorders: a review of the evidence. Gut 2007;56(suppl 1):1-113S.

3 Prospective Studies Collaboration. Body-mass index and causespecific mortality in 900000 adults: collaborative analyses of 57 prospective studies. Lancet 2009;373:1083-96.

4 Hospital Episode Statistics. Homepage. 2010. www.hesonline.nhs. uk. 
5 Kendrick S, Clarke J. The Scottish record linkage system. Health Bull (Edinb) 1993;51:72-9.

6 Allen N, Beral V, Casabonne D, Kan SW, Reeves GK, Brown A, et al. Moderate alcohol intake and cancer incidence in women. J Natl Cancer Inst 2009;101:296-305.

7 Spencer E, Appleby P, Davey G, Key T. Validity of self-reported height and weight in 4808 EPIC-Oxford participants. Public Health Nutr 2002;5:561-5.

8 MacMahon S, Peto R, Cutler J, Collins R, Sorlie P, Neaton J, et al. Blood pressure, stroke, and coronary heart disease. Part 1. Prolonged differences in blood pressure: prospective observational studies corrected for the regression dilution bias. Lancet 1990;335:765-74.

9 Easton D, Peto J, Babiker A. Floating absolute risk: an alternative to relative risk in survival and case-control analysis avoiding an arbitrary reference group. Stat Med 1991;10:1025-35.

10 Collaborative Group on Hormonal Factors in Breast Cancer. Breast cancer and hormonal contraceptives: further results. Contraception 1996;54:1-106S.

11 Townsend $\mathrm{P}$, Phillimore $\mathrm{P}$, Beattie $\mathrm{A}$. Health and deprivation: inequality and the north. Croom Helm, 1988.
12 Klatsky AL, Armstrong MA. Alcohol, smoking, coffee, and cirrhosis. Am J Epidemiol 1992;136:1248-57.

13 Liu B, Balkwill A, Roddam A, Brown A, Beral V. Separate and joint effects of alcohol and smoking on the risks of cirrhosis and gallbladder disease in middle-aged women. Am J Epidemiol 2009;169:153-60.

14 El-Serag HB, Tran T, Everhart J. Diabetes increases the risk of chronic liver disease and hepatocellular carcinoma. Gastroenterology 2004;126:460-8.

15 National Centre for Social Research. Health survey for England 2003. Department of Health, 2004.

16 Office for National Statistics. The national diet and nutrition survey: adults aged 19 to 64 years. Vol 2. HMSO, 2003.

17 Ioannou GN, Weiss NS, Boyko EJ, Kowdley KV, Kahn SE, Carithers RL, et al. Is central obesity associated with cirrhosis-related death or hospitalization? A population-based, cohort study. Clin Gastroenterol Hepatol 2005;3:67-74.

18 Farrell GC, Larter CZ. Nonalcoholic fatty liver disease: from steatosis to cirrhosis. Hepatology 2006;43:99-112S

19 Angulo P. Nonalcoholic fatty liver disease. N Engl J Med 2002;346:1221-31. 